\title{
Spinal intramedullary hemangioblastoma and schwannoma collision tumor: illustrative case
}

\author{
Matthew T. Neal, MD, ${ }^{1}$ Alexandra E. Richards, DNP, FNP-BC, ${ }^{1}$ Kara L. Curley, PA-C,${ }^{1}$ Kliment Donev, MD, ${ }^{2}$ Mark K. Lyons, MD, ${ }^{1}$ and \\ Maziyar A. Kalani, MD ${ }^{1}$
}

1Department of Neurosurgery, Mayo Clinic Arizona, Phoenix, Arizona; and 2Department of Pathology, Mayo Clinic Minnesota, Rochester, Minnesota

\begin{abstract}
BACKGROUND Intramedullary spinal cord tumors represent a minority of intradural tumors. Among intramedullary spinal cord tumors, hemangioblastomas are uncommon, and schwannomas are extremely rare. Collision tumors are histologically distinct tumors that are intermingled and growing together.

OBSERVATIONS In this report, the authors describe a patient with a cervical intramedullary collision tumor involving a hemangioblastoma and schwannoma. To the authors' knowledge, no prior spinal intramedullary collision tumor involving multiple neoplasms has been described. The patient's presentation and management are described.

LESSONS Clinicians should consider the possibility of collision tumors when evaluating intramedullary spinal cord tumors, especially when patient presentation and radiographic findings are atypical. When tumors with similar radiographic characteristics form collision tumors, distinction using preoperative imaging can be extremely challenging. In addition, surgical management of intramedullary collision tumors, like that for all intramedullary spinal cord tumors, should involve meticulous perioperative care and a methodical surgical technique. Maximal safe resection will depend upon histopathological diagnosis, anatomical location of the tumor, presence of distinct dissection planes, and stability of neuromonitoring. Finally, ongoing research on the genetics of intramedullary spinal cord tumors may identify underlying genetic links for intramedullary hemangioblastomas and schwannomas.
\end{abstract}

https://thejns.org/doi/abs/10.3171/CASE2059

KEYWORDS intramedullary spinal cord tumor; schwannoma; hemangioblastoma; collision tumor; spine

Spinal tumors are typically classified as intramedullary, intradural extramedullary, or extradural. Intramedullary spinal cord tumors (IMSCTs) are rare and have diverse pathologies. IMSCTs account for a minority $(20 \%-30 \%)$ of all primary intradural tumors. Within the IMSCT category, gliomas account for $80 \%$ of the tumors. Hemangioblastomas (HBLs) are the third most common tumor type and are estimated to account for $3 \%-8 \%$ of IMSCTs. Other rare tumors, such as intramedullary schwannomas (IMSs), collectively compose the remaining 10\%-12\%. ${ }^{1}$

Spinal intramedullary HBLs are highly vascularized lesions that are considered benign (World Health Organization grade I). ${ }^{2}$ However, they can cause significant morbidity, and even death, depending on mass effect and location. Thirteen percent of HBLs are found within the spinal canal. ${ }^{2}$ The lesions are often solitary, but they may be multifocal in von Hippel-Lindau (VHL) syndrome. The most common age at presentation is 47 years old, ${ }^{3}$ and the tumors occur at the same rate in men and women. ${ }^{4}$ These tumors arise from the pia, but they can have an intramedullary component causing cord expansion. Alternatively, the tumors may grow exophytically from the cord surface or exist entirely in the extramedullary space. The predominant location is dorsal and intramedullary with presentation to the cord surface..$^{1,3}$

Schwannomas arise from Schwann cells, the myelin-producing cells of the peripheral nervous system. Histologically, the majority of schwannomas are benign. However, spinal schwannomas can also cause significant symptomatology from compression of the nerve roots and/or the spinal cord. Spinal schwannomas most commonly arise from cranial and spinal nerves, although they can be found in the intramedullary compartment. IMSs are rare, representing $1.1 \%$ of spinal schwannomas. ${ }^{5}$ A recent systematic review identified 166 cases in the literature. ${ }^{6}$ Spinal schwannomas are solitary and sporadic $90 \%$ of the

ABBREVIATIONS GTR = gross-total resection; $\mathrm{HBL}=$ hemangioblastoma; $\mathrm{HIF1a}=$ hypoxia-inducible factor $1 \mathrm{a} ; \mathrm{IMS}=$ intramedullary schwannoma; $\mathrm{IMSCT}=$ intramedullary spinal cord tumor; $\mathrm{MRI}$ = magnetic resonance imaging; $\mathrm{NF2}$ = neurofibromin 2; STIR = short tau inversion recovery; $\mathrm{T} 1 \mathrm{WI}=\mathrm{T} 1$-weighted imaging; $\mathrm{T} 2 \mathrm{WI}=\mathrm{T} 2$-weighted imaging; $\mathrm{VHL}=$ von Hippel-Lindau.

INCLUDE WHEN CITING Published January 18, 2021; DOI: 10.3171/CASE2059.

SUBMITTED October 9, 2020. ACCEPTED October 26, 2020.

(C) 2021 The authors, CC BY-NC-ND 4.0 (http://creativecommons.org/licenses/by-nc-nd/4.0/). 
time. Multiple schwannomas raise the suspicion for an underlying disorder such as neurofibromatosis type 2 or schwannomatosis. ${ }^{4}$ Spinal schwannomas are most commonly seen in individuals aged 65-74 years, with males more commonly affected than females. ${ }^{7}$

Collision tumors are defined as "histologically distinct tumors arising at independent topographical sites, simultaneously growing, meeting together, and eventually intermingling."8 Although there are a limited number of case reports describing collision tumors within the intracranial and spinal compartments, the authors are not aware of any reported spinal intramedullary collision tumors involving 2 distinct neoplasms. ${ }^{9-11}$ In this report, we present a unique case with a cervical intramedullary HBL and IMS collision tumor. Patient presentation, management, and lessons learned are discussed.

\section{Illustrative Case}

The patient was a 51-year-old woman with a medical history significant only for hyperlipidemia, who presented with a 2-year history of nondermatomal numbness distal to the elbow and fine motor control difficulty of the right upper extremity. She also described a curling sensation in her toes. She had an intact motor and sensory examination finding. However, she had 4+ reflexes in her upper and lower extremities. Magnetic resonance imaging (MRI) revealed increased T2 signal within the cervical spinal cord spanning from the foramen magnum down to the upper thoracic spine. Postcontrast imaging demonstrated a $5 \times 5 \times 10$-mm contrast-enhancing intramedullary mass in the dorsal aspect of the spinal cord at C4. There was also a linear focus of low T2 signal and short tau inversion recovery (STIR) signal within the mass consistent with remote hemorrhage (Figs. 1 and 2). No suspicious contrast-enhancing lesions were identified on imaging of the remainder of the neural axis.

Surgery was recommended to obtain a pathological diagnosis and to reduce tumor volume. Although decompression alone was considered for

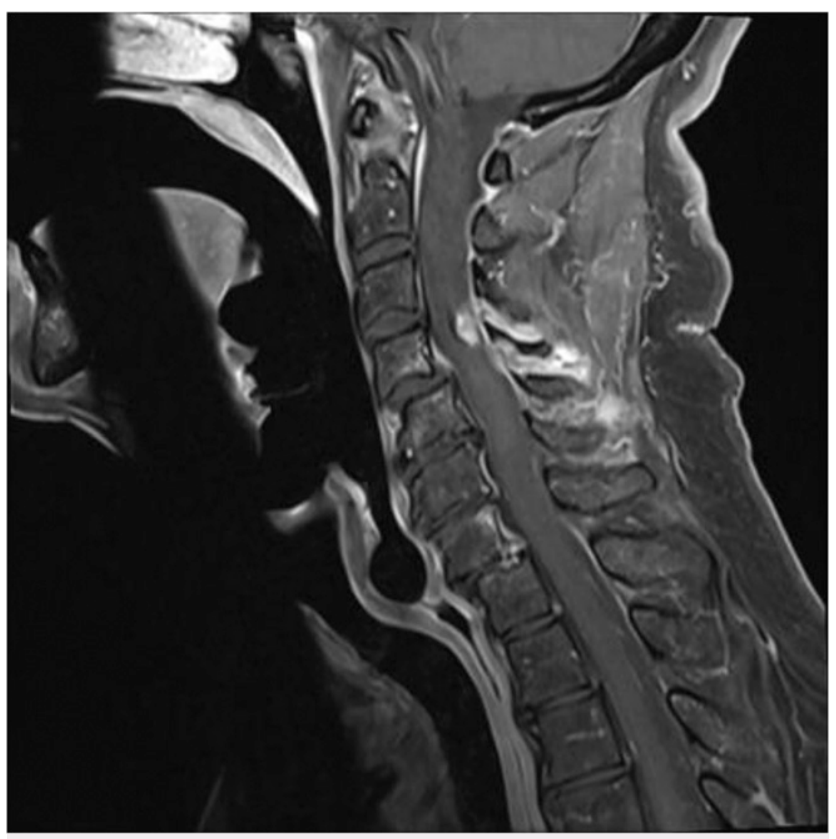

FIG. 1. Contrast-enhanced sagittal T1 MRI demonstrating an avidly enhancing intramedullary spinal tumor at the C4 level.

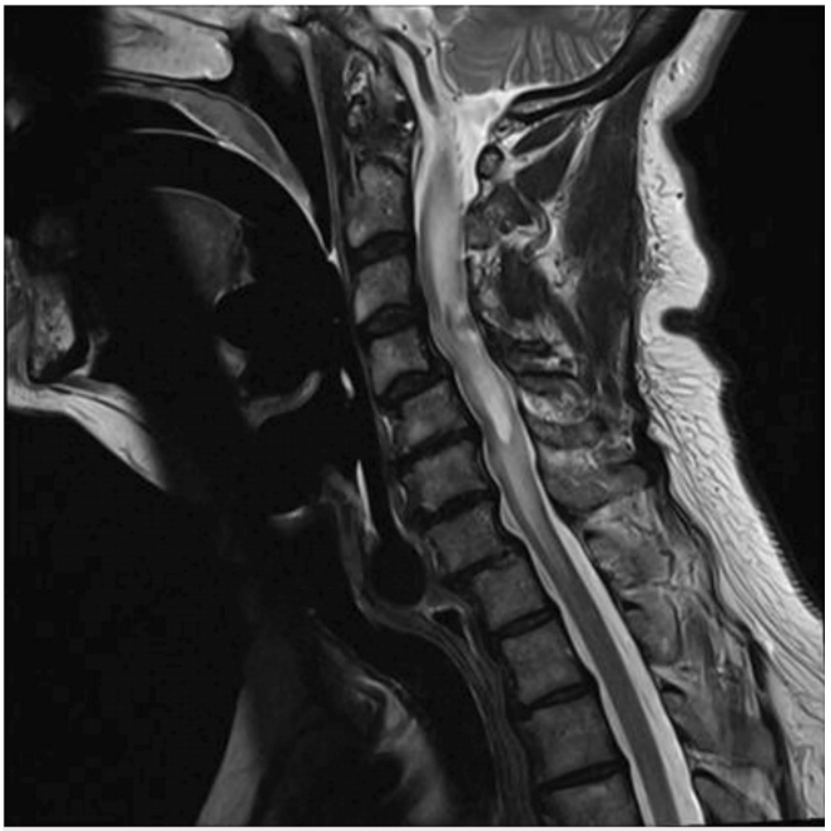

FIG. 2. Sagittal T2 MRI demonstrating a hypointense intramedullary tumor at $\mathrm{C} 4$ and extensive surrounding edema.

the patient, MRI demonstrated subluxation with instability at C4-C5, so the patient was a candidate for a decompression and instrumented fusion. The patient elected to pursue surgery. Cervical 3-5 laminectomies were performed. A midline durotomy was performed. There was a notable area of hemosiderin staining and splaying of the posterior columns where the tumor presented to the dorsal surface of the spinal cord. A combination of focused bipolar cautery, microscissors, and Rhoton microdissector instruments was used to dissect and shrink the tumor circumferentially. Dissection at the cephalad and caudal poles was facilitated by cerebrospinal fluid clefts. The deep draining veins were coagulated and cut, allowing en bloc removal of the tumor. Frozen pathology of the specimen was most consistent with schwannoma. Once the tumor was removed, motor evoked potentials were improved in the right upper extremity, and somatosensory signals were stable. Following closure of the dura, a posterolateral fusion was performed using morselized autograft and C3-C5 lateral mass screw and rod fixation.

Microscopic examination revealed a low-grade tumor composed of 2 morphologically different components. One component consisted of uniform spindle cells arranged in interlacing fascicles (Fig. 3), and the second component consisted of epithelioid foamy stromal cells and a capillary network (Fig. 4). Spindle cells were highlighted by $\mathrm{S} 100$, collagen 4, and SOX10 immunohistochemical stains, whereas foamy cells were weakly positive for neuron-specific enolase and S100 and negative for SOX10 and inhibin. Glial fibrillary acidic protein and CD68 were negative on both components. The morphological features and immunoprofile were consistent with schwannoma and HBL collision tumor.

Six months following surgery, the patient's neurological examination finding was stable. MRI revealed marked improvement in the preoperative syrinx and spinal cord edema (Fig. 5). Contrast-enhanced images did not reveal any evidence of tumor recurrence. Cervical radiographs demonstrated a stable fusion construct. 


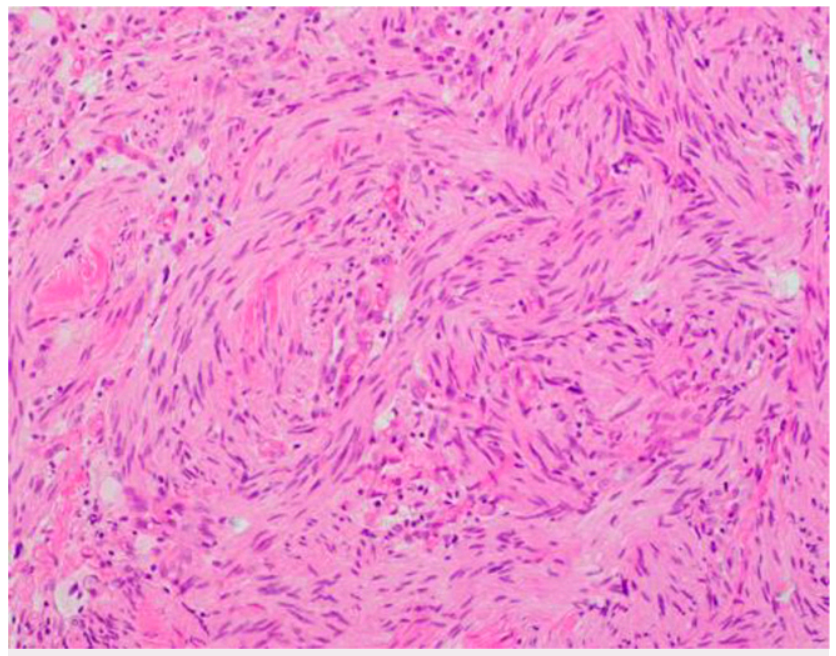

FIG. 3. Schwannoma demonstrating uniform spindle cells arranged in interlacing fascicles (Antoni A pattern) (hematoxylin-eosin stain, original magnification $\times 400)$.

\section{Discussion}

\section{Observations}

The patient in this case presented with a unique spinal intramedullary collision tumor involving 2 nonglial tumors: an $\mathrm{HBL}$ and an IMS. Rare intracranial collision tumors involving $\mathrm{HBL}$ with ependymoma ${ }^{12}$ and chordoma have been reported..$^{13}$ Collision tumor case reports have also demonstrated intracranial schwannomas intermingled with extracranial metastases, meningiomas, pituitary adenomas, and plasma cell tumors. . $^{811,14-16}$ In the spinal compartment, 2 collision tumor cases have been reported. One involved an intradural extramedullary astrocytoma and ependymoma, ${ }^{17}$ and the other involved an IMS with an intramedullary cysticercus. ${ }^{18} \mathrm{~A}$ spinal

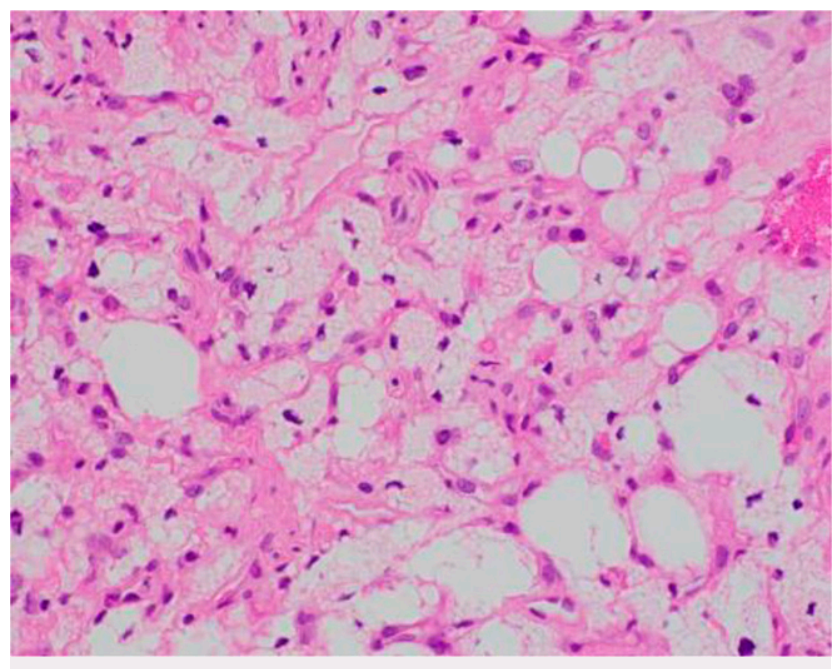

FIG. 4. HBL component with foamy stromal cells and capillary vascular channels (hematoxylin-eosin stain, original magnification $\times 400)$.

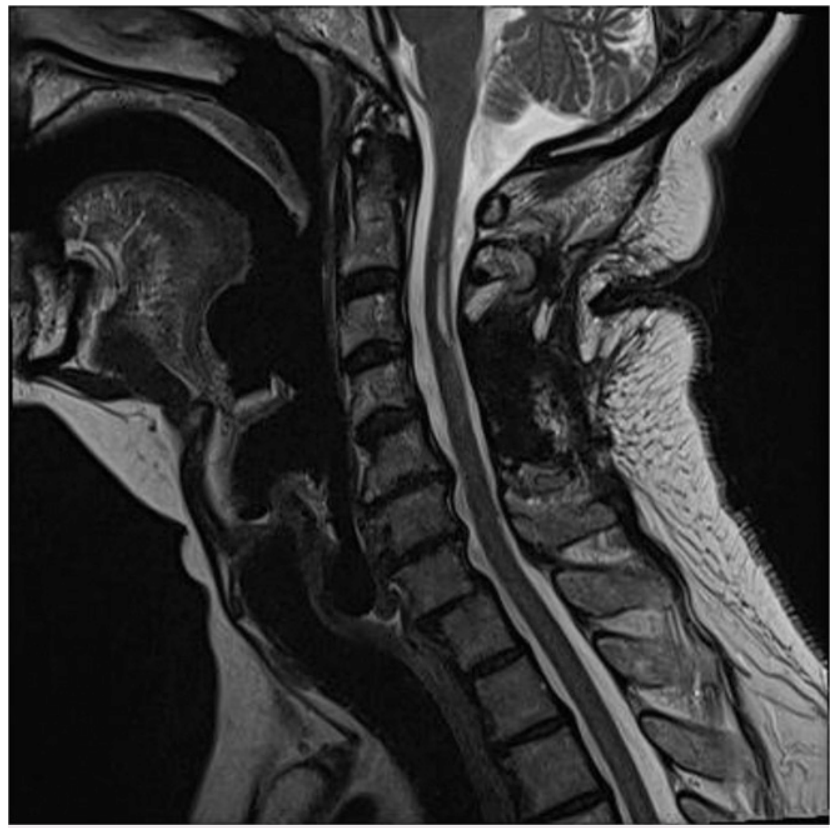

FIG. 5. Sagittal T2 MRI 4 weeks following surgery demonstrating marked improvement in the syrinx and cord edema.

intramedullary collision tumor involving 2 distinct neoplastic tumors, such as the one in the present case, has not been previously reported.

The clinical manifestations of IMSCTs will vary on the basis of anatomical location and associated factors such as a syrinx and peritumoral edema. The patient in this case presented with a 2-year progressive history of myelopathy affecting the upper and lower extremities. Although the tumor was dorsal in location and limited in size, the patient had significant dysfunction related to the extensive edema and syrinx within the cord, likely related to arteriovenous shunting or venous congestion.

In addition to the cervical location and perilesional edema out of proportion to the tumor size, the tumor in this case had other radiographic findings consistent with HBL. Similar to the tumor in this case, HBLs are typically iso- to hypointense on T1-weighted imaging (T1WI) sequences and hyperintense on T2-weighted imaging (T2WI) sequences. Smaller lesions typically have homogeneous, avid enhancement. Another differentiating feature of HBLs is hemorrhage. ${ }^{1}$ In this case, there was also a linear focus of low T2 signal and STIR signal within the mass consistent with remote hemorrhage. The dorsal location with presentation to the cortical surface, as in this case, is also common for HBL. ${ }^{1}$ Flow voids on T2WI, commonly seen with larger lesions, were not noted in this case.

Adding to the preoperative diagnostic dilemma in this case, IMSs have radiographic features that overlap with those of HBLs. Similar to $\mathrm{HBLs}$, IMSs are usually well-encapsulated solid masses that are iso- to hypointense on T1WI and hyperintense on T2WI. The patient in this case did have an adjacent cystic cavity in proximity to the enhancing mass, which is consistent with both HBLs and IMSs. The patient in this case did not have an intramedullary component extending into the extramedullary space and connecting with a thickened nerve root, which is pathognomonic for IMS.6,19

We recommended surgery in this case to obtain a diagnostic specimen, alleviate tumor mass effect and surrounding edema, and 
perform a maximal safe resection. We methodically planned the surgical procedure. Preoperative embolization was contemplated, but it was not used, owing to the small size and cervical location of the tumor. Intraoperatively, the surgical team maintained close communication with the multidisciplinary team, including anesthesia, pathology, and intraoperative neuromonitoring personnel. The anesthesia team strictly maintained adequate mean arterial pressures.

We used redundant strategies to localize the tumor. We confirmed our laminectomy sites using fluoroscopy. Once the laminectomies were complete, we confirmed tumor location using ultrasonography. Following the midline durotomy, we were prepared to perform posterior column mapping for identification of the safe entry zone. However, color changes on the dorsal surface of the spinal cord precisely identified the location of the tumor. Once the tumor was encountered, an early frozen section was consistent with IMS, and we elected to proceed with maximal safe resection. Studies have shown that gross-total resection (GTR) is possible $83 \%$ of the time with spinal schwannomas. ${ }^{4,6} \mathrm{HBLs}$ in the spinal cord also have high rates of GTR $(93 \%-99 \%) .{ }^{3}$ Dissection in this case was facilitated by the discernable interface between the tumor and the spinal cord parenchyma. We also paid close attention to motor and somatosensory signals during dissection.

No adjuvant treatment, such as spinal radiosurgery, has been done because GTR was accomplished. The patient is undergoing close radiographic and clinical follow-up. Spinal HBLs have a favorable functional stability rate $(86 \%)$ at 5 years. Recurrence rates following GTR of sporadic, non-VHL-associated spinal HBLs is less than $7 \%{ }^{3}$ Similarly, spinal schwannomas have low recurrence rates, less than $5 \%$, following GTR. ${ }^{20}$

As previously mentioned, HBLs are one of the more common (3\%-8\%) types of IMSCT. Twenty-five percent of spinal HBLs are associated with $\mathrm{VHL}$ disease, characterized by the $\mathrm{VHL}$ mutation. The $V H L$ gene encodes an E3 ubiquitin ligase that targets hypoxia-inducible factor 1a (HIF1a). HIF1a regulates cell metabolism and vascular proliferation. Other than the $\mathrm{VHL}$ gene, understanding of the genetic basis for spinal HBL remains limited. ${ }^{4,21}$

IMSs are much less common than spinal HBLs, presumably due to the lack of Schwann cells in the spinal cord. There have been multiple hypotheses about the origin of IMS. Authors have proposed that IMS may arise from Schwann cells on small peripheral nerves that run alongside intraspinal vessels. Neural crest cell dislocation into the parenchyma during embryogenesis and metaplasia of pial cells leading to the development of IMS are alternative hypotheses. ${ }^{5,6}$

Mutations in the neurofibromin 2 (NF2) gene, located on chromosome 22q12.2, can lead to schwannomas in the spine. The NF2 protein is a member of the ERM (ezrin, radixin, and moesin) family of proteins, which links cytoskeletal components and proteins of the cell membrane that regulate cytoskeletal dynamics and cell-to-cell communication. In addition, mutations in INI1/SMARB1, a gene involved in chromatin remodeling, have been observed in familial schwannomatosis.,21 Similar to spinal $\mathrm{HBL}$, several genetic alterations leading to tumorigenesis have been identified, but a comprehensive understanding remains incomplete. Currently, there is no known genetic basis for the co-occurrence of intramedullary HBL and IMS.

\section{Lessons}

Clinicians should consider the possibility of collision tumors when evaluating IMSCTs, especially when patient presentation and radiographic findings are atypical. When tumors with similar radiographic characteristics form collision tumors, distinction using preoperative imaging can be extremely challenging. In addition, surgical management of intramedullary collision tumors, as with all IMSCTs, should involve meticulous perioperative care and a methodical surgical technique. Maximal safe resection will depend upon histopathological diagnosis, anatomical location of the tumor, presence of distinct dissection planes, and stability of neuromonitoring. Finally, ongoing research on the genetics of IMSCTs may identify an underlying genetic link for intramedullary HBLs and IMSs.

This case involves a unique intramedullary spinal collision tumor containing HBL and IMS. It is unknown if there is an underlying genetic link that led to the formation of the collision tumor. The tumor in this case was successfully managed using a standardized approach for IMSCT, despite the presence of 2 tumor types.

\section{References}

1. Kandemirli SG, Reddy $A$, Hitchon $P$, et al. Intramedullary tumours and tumour mimics. Clin Radiol. 2020;75(11):876.e17-876.e32.

2. Tuleasca $C$, Knafo $S$, David $P$, et al. A rare condition: spontaneous subarachnoid hemorrhage due to spinal hemangioblastoma: report of 2 cases and review of the literature. Neurochirurgie. 2020; 66(5):359-364.

3. Hussain I, Parker WE, Barzilai O, et al. Surgical management of intramedullary spinal cord tumors. Neurosurg Clin N Am. 2020; 31(2):237-249.

4. Azad TD, Jiang B, Bettegowda C. Molecular foundations of primary spinal tumors-implications for surgical management. Ann Transl Med. 2019;7(10):222

5. Landi A, Grasso G, Gregori F, et al. Isolated pediatric intramedullary schwannoma: case report and review of literature. World Neurosurg. 2018;115:417-420.

6. Colosimo C, Cerase A, Denaro L, et al. Magnetic resonance imaging of intramedullary spinal cord schwannomas. Report of two cases and review of the literature. J Neurosurg. 2003;99(1 Suppl):114-117.

7. Tish S, Habboub G, Lang M, et al. The epidemiology of spinal schwannoma in the United States between 2006 and 2014. J Neurosurg Spine. 2019;32(5):1-6.

8. Adib SD, Tatagiba M. Surgical management of collision-tumors between vestibular schwannoma and meningioma in the cerebellopontine angle in patients with neurofibromatosis type 2 Acta Neurochir (Wien). 2019;161(6):1157-1163.

9. Bougaci N, Litrico S, Burel-Vandenbos F, et al. Unusual cauda equina syndrome due to multifocal ependymoma infiltrated by lymphoma. J Spine Surg. 2017;3(4):697-701.

10. Blue R, Yang Al, Zhou C, et al. Telemedicine in the era of coronavirus disease 2019 (COVID-19): a neurosurgical perspective. World Neurosurg. 2020;139:549-557.

11. Tang GC, Piao YS, Zhao L, et al. Lung adenocarcinoma metastasizing to cerebellopontine angle schwannoma (collision tumor). Acta Neurochir (Wien). 2007;149(1):87-90.

12. Schild MH, Doane EP, Friedman AH, et al. Mixed hemangioblastoma and ependymoma collision tumor of the cerebellum. Clin Neuropathol. 2017;36(5):248-249.

13. Fukaya T, Yoshida J, Banno T, et al. Intracranial collision tumor -a case report. Article in Japanese. No Shinkei Geka. 1976;4(6): 585-591.

14. Yang MJ, Arkun K, Heilman CB. Tumor-to-tumor metastasis of hepatocellular carcinoma to vestibular schwannoma. World Neurosurg. 2019;128:324-327.

15. Bakhit MS, Fujii M, Jinguji S, et al. Jugular foramen collision tumor (schwannoma and plasma cell pseudotumor), a probable lgG4 related disease. World Neurosurg. 2017;102:694.e9-694.e13.

16. Koutourousiou M, Kontogeorgos $G$, Wesseling $P$, et al. Collision sellar lesions: experience with eight cases and review of the literature. Pituitary. 2010;13(1):8-17. 
17. Weinstein GM, Arkun K, Kryzanski J, et al. Spinal intradural, extramedullary ependymoma with astrocytoma component: a case report and review of the literature. Case Rep Pathol. 2016;3534791.

18. Vij M, Jaiswal $S$, Jaiswal $A K$, et al. Coexisting intramedullary schwannoma with intramedullary cysticercus: report of an unusual collision. Indian J Pathol Microbiol. 2011;54(4):866-867.

19. Riffaud L, Morandi X, Massengo S, et al. MRI of intramedullary spinal schwannomas: case report and review of the literature. Neuroradiology. 2000;42(4):275-279.

20. Fehlings MG, Nater A, Zamorano JJ, et al. Risk factors for recurrence of surgically treated conventional spinal schwannomas: analysis of 169 patients from a multicenter international database. Spine (Phila Pa 1976). 2016;41(5):390-398.

21. Karsy M, Guan J, Sivakumar W, et al. The genetic basis of intradural spinal tumors and its impact on clinical treatment. Neurosurg Focus. 2015;39(2):E3.

\section{Disclosures}

Dr. Kalani does general consulting for Medtronic, Nuvasive, and CarboFix Orthopedics.

\section{Author Contributions}

Conception and design: Neal, Richards, Kalani. Acquisition of data: Neal, Lyons, Kalani. Analysis and interpretation of data: Donev, Lyons, Kalani. Drafting the article: Neal, Richards, Curley, Lyons. Critically revising the article: Neal, Richards, Curley, Lyons, Kalani. Reviewed submitted version of manuscript: all authors. Approved the final version of the manuscript on behalf of all authors: Neal. Administrative/technical/ material support: Curley, Lyons. Study supervision: Neal, Lyons, Kalani.

\section{Correspondence}

Matthew T. Neal: Mayo Clinic, Phoenix, AZ. neal.matthew@ mayo.edu. 